\title{
Niederschlags-Abfluss-Modellierung im urbanen Raum
}

\author{
Gerald Krebs (iD
}

Online publiziert: 12. Mai 2020

(C) Der/die Autor(en) 2020

Zusammenfassung Hydrologische Simulationen sind ein adäquates Mittel, um wasserwirtschaftliche Maßnahmen im urbanen Raum zu planen und deren Wirkungsweise $\mathrm{zu}$ evaluieren. Dieser Beitrag beschreibt die Erstellung eines Niederschlags-Abfluss-Modells für den urbanen Raum durch die Verwendung von Open-Source-Software. Das Stormwater Management Model (SWMM) dient als Simulationssoftware, die Modellerstellung erfolgt mit Open-SourceGIS-Software und die Kalibrierung und Nachbereitung läuft über Pakete in $\mathrm{R}$ und Python. Abschließend wird der gesamte Modellaufbau an einem Anwendungsbeispiel erläutert.

Schlüsselwörter Stormwater

Management Model .

Modellerstellung · Urbane Hydrologie . Datenbedarf und -verfügbarkeit

\section{Rainfall-runoff modelling in urban areas}

Abstract Hydrological simulations are an adequate tool to design and evaluate stormwater management measures in urban areas. This contribution outlines the development and setup of a rainfall runoff model using open source software. Hydrological simulations are conducted using the Stormwater Management Model (SWMM), open source GIS software is used for the model setup, and model calibration and validation as well as data handling are conducted using $\mathrm{R}$ and Python packages. Finally, the setup process is discussed using a real application.

Keywords Stormwater Management Model · Model setup · Urban hydrology $\cdot$ Data requirements and availability

DI Dr. G. Krebs ( $\square)$ Institut für Wasserbau und Wasserwirtschaft, Technische Universität Graz, Stremayrgasse 10/II, $8010 \mathrm{Graz}$, Österreich gerald.krebs@tugraz.at

\section{Einleitung}

Die Urbanisierung in Zusammenspiel mit demografischen Veränderungen und dem Klimawandel stellt die Wasserwirtschaft allgemein und speziell in urbanen Gebieten vor große Herausforderungen. Hierbei geht es auf der einen Seite um die Sicherung der Wasserversorgung und auf der anderen Seite um nachhaltigere und flexiblere Ansätze zur Entwässerung von Städten. Das Hauptaugenmerk hierbei liegt vor allem auf dem Umgang mit Regenwasser in der Stadt.

Eine Zunahme an versiegelten Flächen erhöht den Oberflächenabfluss, während sowohl Versickerung als auch Verdunstung abnehmen (Leopold 1968; Schueler et al. 2009). Erhöhter Oberflächenabfluss führt zu einer häufigeren und stärkeren Belastung des Kanalnetzes und damit zum Eintrag von Schadstoffen in Oberflächengewässer durch Mischwasserentlastungen. Durch häufigere Überlastungen des Kanalnetzes zum einen durch fortschreitende Versiegelung und zum anderen durch eine Zunahme von Starkniederschlagsereignissen - ist auch ein Anstieg an Überflutungen $\mathrm{zu}$ beobachten (Huong und Pathirana 2013). Durch den steigenden Versiegelungsgrad nimmt die Versickerung und damit die Anreicherung des Grundwassers ab (Haase und Nuissl 2007). Gleichzeitig steigt durch die fehlende Verdunstung die Lufttemperatur in der Stadt und es kommt zur Ausbildung von lokalen Hitzeinseln (Wilby 2008). Diese Auswirkungen erfordern eine Anpassung der wasserwirtschaftlichen Strategie in urbanen Räumen. Mögliche Maßnahmen reichen von naturnahen Systemen (z.B. Gründächer, Entsiegelung, Mulden), die Versickerung und/oder Verdunstung fördern, über die Vermeidung von Versiegelung schon im Planungsstadium bis zu semizentralen bzw. zentralen Maßnahmen. Vor- und Nachteile der Systemansätze sind dabei im Detail abzuwägen.

Zur Evaluierung unterschiedlicher Strategien und Maßnahmen ist die hydrologisch-hydrodynamische Model- lierung ein adäquates Mittel. Mithilfe von Simulationen können unterschiedlichste Belastungsfälle und Szenarien evaluiert werden - von der Entwicklungs- bis zur Detailplanung. Dazu steht eine breite Auswahl an Werkzeugen zur Verfügung, die sich in Verfügbarkeit, Simulationsansätzen und Schwerpunkten unterscheiden (Zoppou 2001; Singh und Woolhiser 2002). Genannt seien hier, da es um die urbane Anwendung geht, MIKE-URBAN (DHI) und das Stormwater Management Model (SWMM), die mit zu den vollständigsten und meistverwendeten Modellen gehören. Neben einer Vielzahl an proprietären Varianten (z.B. PCSWMM, InfoSWMM) wird SWMM von der US Environmental Agency als Open-Source-Paket zur Verfügung gestellt.

Die Wahl eines geeigneten Modells hängt maßgeblich von den Zielen der Simulation ab. Generell beinhaltet eine Modellierungsaufgabe aber immer folgende Schritte: (i) Aufbereitung der hydro-meteorologischen und räumlichen Daten, (ii) Erstellung des Modells, (iii) Nachbearbeitung und Kalibrierung bzw. Validierung. Kommerzielle Plattformen bieten die notwendigen Tools für diese Schritte oft in einem Paket. Will oder muss man hingegen auf Open-Source-Plattformen zurückgreifen, ist in den meisten Fällen die Kombination verschiedener Softwarepakete nötig.

In diesem Beitrag werden die Schritte zur Erstellung eines hydrologischen Modells für die urbane Wasserwirtschaft, unter der Verwendung von Open-Source-Software, beschrieben und erörtert. Die Schritte umfassen die Aufbereitung räumlicher Daten mittels Open-Source-GIS-Software, die Modellerstellung für SWMM und die Kalibrierung bzw. Nachbearbeitung mit R bzw. Python. Abschließend wird der Ablauf anhand eines umgesetzten Beispiels diskutiert. 


\section{Stormwater Management Model (SWMM)}

Das Stormwater Management Model SWMM wurde 1971 von der US Environmental Protection Agency (EPA) entwickelt und seitdem kontinuierlich weiterentwickelt und erweitert. SWMM wurde spezifisch auf die urbane Niederschlags-Abfluss-Modellierung abgestimmt und erlaubt die Simulation von Abflussprozessen sowohl in Bezug auf die Wassermenge als auch auf die Wasserqualität (Huber und Dickinson 1988; Rossman 2015).

SWMM bildet die Entwässerungsprozesse mithilfe einer Reihe gekoppelter, weiter unterteilter Module ab. Im Atmosphärenmodul werden der Niederschlag und klimatologische Randbedingungen definiert und berechnet. Das Oberflächenmodul generiert über Verlustansätze Abfluss, der an das Grundwasser- bzw. das Transportmodul weitergegeben wird. Das Transportmodul bildet das eigentliche Entwässerungssystem über Rohrleitungen und offene Gerinne ab. SWMM-Berechnungen basieren auf nichtlinearen Speicherkaskaden, die Zufluss durch Niederschlag und von benachbarten Teilflächen erhalten und Abfluss, Versickerung und Verdunstung generieren. Die Speicherkapazität wird definiert durch die Oberflächenbeschaffenheit (Mulden und Interzeption). Wenn das vorhandene Oberflächenwasser die Speicherkapazität übersteigt, kommt es zum Oberflächenabfluss, der mit dem Ansatz von Manning berechnet wird.

Die Einzugsgebietsfläche wird in möglichst hydrologisch homogene Teilflächen diskretisiert, deren Versiegelungsgrad den durchlässigen und undurchlässigen Anteil definiert. Während Verdunstung auf der gesamten Teilfläche stattfindet, kann Versickerung nur auf dem durchlässigen Anteil stattfinden (Rossman 2015). Für die Berechnung der Versickerung stehen neben den Ansätzen nach Horton und GreenAmpt (Rawls et al. 1992) auch die vor allem in den USA gebräuchliche Curve Number (CN) Methode und einfache Verlustansätze zur Verfügung.

Der Abfluss im Entwässerungssystem kann mit simplifizierten Ansätzen oder dynamisch gelöst werden. Im dynamischen Ansatz wird die vollständige Saint-Venant-Gleichung eindimensional gelöst. Der Zusammenhang zwischen Abfluss, Wassertiefe und Reibungsverlust wird dabei über die Gleichung nach Manning hergestellt (Rossman 2015).

\section{Datenbedarf und -verfügbarkeit}

Der Bedarf an räumlichen und hydrometeorologischen Daten wird, wie die Wahl des Modells, maßgeblich durch die Ziele der Modellierungsaufgabe und die Beschaffenheit des zu modellierenden Gebiets bestimmt. Während Oberflächeninformationen wie Landnutzung und Versiegelung immer Relevanz haben, werden Daten zu den Bodeneigenschaften bei Gebieten mit sehr hohen Versiegelungsgraden oft nicht benötigt. Geometrische Randbedingungen wie Bruchkanten, Fließwege und Kanaleinläufe haben bei urbanen Gebieten hingegen immer besondere Bedeutung. Grundsätzlich kann festgestellt werden, dass gute Informationen zum Einzugsgebiet aussagekräftige Simulationsergebnisse unterstützen, Unsicherheiten reduzieren und den Modellaufbau erleichtern.

Die Verfügbarkeit räumlicher Daten mit hinreichenden Informationen verbessert sich stetig. Versiegelungskarten und Kanalkataster sind in den meisten Städten bereits digitaler Standard. Bei Detailinformationen wie Bruchkanten und Oberflächenbelägen sieht die Situation meist schlechter aus. Allerdings gibt es auch hier dank höher auflösender digitaler Höhenmodelle und Satellitendaten Verbesserungen. Genaue digitale Aufnahmen der Bruchkanten und Oberflächen sind zurzeit aber noch die Ausnahme.

Hydro-meteorologische Daten umfassen Niederschlagsdaten, klimatologische Informationen und Abflussmessungen. Letztere sind vor allem für die Modellkalibrierung wichtig. Niederschlagsdaten sollten in einer möglichst hohen zeitlichen und räumlichen Auflösung zur Verfügung stehen. Durch die komplexe, feingliedrige Struktur urbaner Gebiete gilt dies unabhängig vom Ziel der Modellierungsaufgabe. Als Richtwert dient hier ein Aufzeichnungsintervall $<5$ min (Schilling 1991). Die räumliche Auflösung entspricht in den meisten Fällen noch nicht dem Bedarf, hier wäre eine räumliche $\mathrm{Ab}$ deckung von einer Messstation je $\mathrm{km}^{2}$ wünschenswert (Cristiano et al. 2017; Berndtsson und Niemczynowicz 1988).

Vor allem in urbanen Gebieten nimmt die Verfügbarkeit von Niederschlags- und Klimadaten laufend $\mathrm{zu}$. Als Beispiel sei hier die Stadt Graz (Ös- terreich) genannt, wo aktuell $21 \mathrm{Nie}$ derschlagswaagen auf das Stadtgebiet verteilt sind. Allerdings zeigt sich auch hier, dass noch dichtere Verteilungen für die Abbildung konvektiver Niederschläge notwendig sind (Maier et al. 2020).

\section{Methodik und Aufbau}

Zur Erstellung eines hydrologisch-hydrodynamischen Modells in SWMM werden Informationen zu Einzugsgebietsoberfläche und Topografie und zum Kanalnetz - Knoten und Haltungen - benötigt. Je nach Modellierungsaufgabe und Betrachtungsgebiet können noch weitere Informationen benötigt werden. Dazu gehören Informationen zu grüner Infrastruktur, Reinigungsprozesse und -intervalle bei der Schmutzfrachtsimulation oder Grundwasserdaten bei seichten Grundwasserkörpern. Der erste Schritt der Modellierungsaufgabe, nachdem die entsprechende Software festgelegt wurde, ist die Analyse vorhandener Datensätze und deren Beschaffung. Dazu zählen neben Versiegelungskarten, Landnutzungsdaten, Gebäude- und Straßenkataster und dem Kanalkataster auch Informationen zu Sonderbauwerken und Einwohnerkennwerte. Weiters benötigt man Niederschlags- und Klimadaten, die das Modell antreiben.

Bevor eine SWMM-Input-Datei erstellt werden kann, müssen die Daten entsprechend aufbereitet werden. Zur Aufbereitung räumlicher Daten eignen sich geografische Informationssysteme (GIS), in denen Objekteigenschaften mit räumlicher Information verknüpft werden. Als Open-Source-Softwarepaket steht hier z.B. QGIS (QGIS Entwicklungsteam 2020; www.qgis.org) zur Verfügung. QGIS bietet Werkzeuge für die gängigen hydrologischen Anwendungen. Einschränkungen im Vergleich zu kommerziellen Produkten wie z.B. ARCGIS (Environmental Systems Research Institute (ESRI) 2012) gibt es zwar zum Teil, diese wirken sich bei der Modellerstellung allerdings nicht negativ aus.

Bei der Aufbereitung der Eingangsdaten werden möglichst alle relevanten Informationen verknüpft und verschnitten, um sowohl für die Oberfläche als auch für das Entwässerungsnetz alle verfügbaren Informationen im Modell zu berücksichtigen. Es empfiehlt sich dabei, alle Informationen in letztendlich drei Datensätze $(s u b$ - 


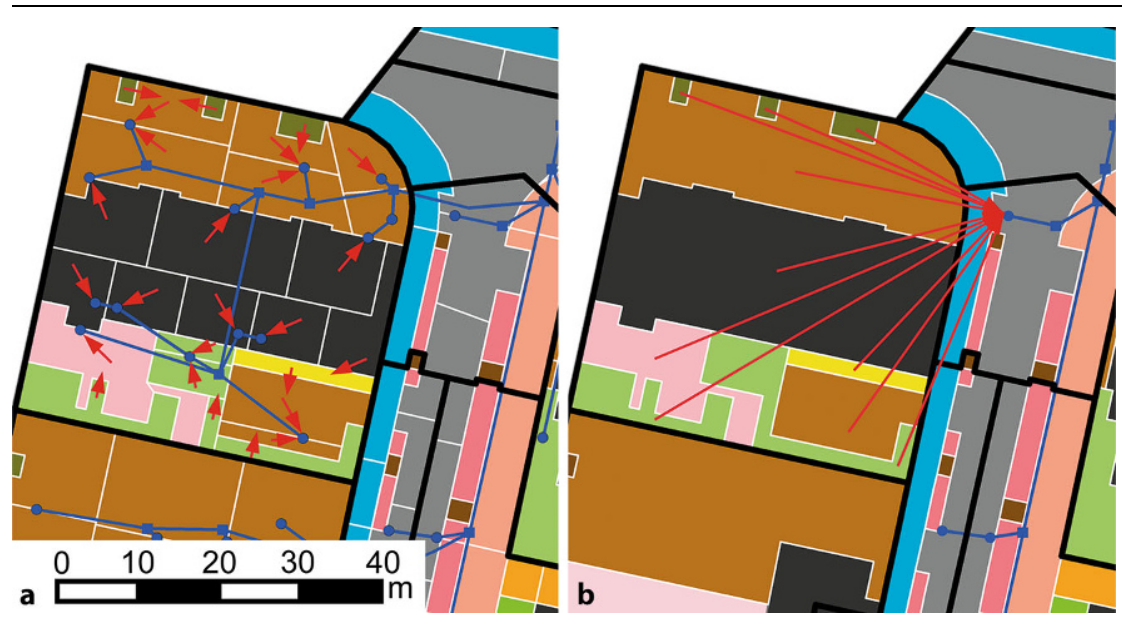

Abb. 1 Leitung des Oberflächenabflusses über Einzelflächen (a) und vereinfacht direkt zum Knoten (b). Die einzelnen Farben beschreiben unterschiedliche Oberflächen, schwarze Linien beschreiben die knotenbezogenen Teileinzugsgebiete und blaue Linien und Punkte beschreiben das Entwässerungsnetz (modifiziert von Krebs 2016)

catchments, nodes, und links) zu übertragen. Die Oberfläche des Einzugsgebietes wird in SWMM im Layer subcatchments hinterlegt. Dieser Layer enthält sowohl alle relevanten Oberflächeninformationen und assoziierte Parameter als auch die Verknüpfung mit dem Entwässerungssystem über den definierten Auslass für jedes Teileinzugsgebiet. Dieser Auslass ist entweder ein Knoten des Entwässerungsnetzes oder ein Teileinzugsgebiet. Der Layer nodes enthält Informationen zu den Knoten, die, über den Anfangsbzw. Endknoten der Haltung, mit dem Haltungslayer links verknüpft sind.

Kanalknoten bzw. Einlässe sind in den meisten Fällen im Kanalkataster hinreichend genau und mit allen relevanten Informationen dokumentiert. Die häufig fehlende maximale Einstauhöhe des Einlasses kann über die Sohlhöhe und den entsprechenden Höhenwert der digitalen Geländemodelle (DGM) ermittelt werden. Ähnliches gilt für Kanalhaltungen. Falls notwendig, müssen die Anfangs- und Endknoten der Haltung definiert werden.

Die Teileinzugsgebiete sollten aus möglichst homogenen Oberflächen bestehen, um die Parametrisierung zu erleichtern. Dazu wird das Projektgebiet in Dachflächen, Straßen- und Bewegungsflächen und Grünflächen nach unterschiedlicher Vegetation aufgeteilt und hydrologische Parameter (z.B. Muldenspeicher, Bodenkennwerte und Rauigkeiten) zugeteilt.

Die Zuteilung der Teileinzugsgebiete kann über ein DGM erfolgen. Da diese Modelle im urbanen Gebiet allerdings einige abflussrelevante Details nicht abbilden, muss zunächst eine Bearbeitung erfolgen, um die Beschreibung des urbanen Geländes und des Entwässerungssystems zu verbessern. Diese Bearbeitung erfolgt rasterbasiert, da es sich bei DGMs auch um Rasterdatensätze handelt und die Verarbeitung einfach ist. Die Arbeitsschritte hängen von der Datengrundlage $a b$, daher sollen hier nur Beispiele gegeben werden. Da das urbane Entwässerungsnetz meist unterirdisch verläuft, ist es im DGM nicht abgebildet. Um im Modell korrekte Fließwege darzustellen, muss es aber berücksichtigt werden. Daher wird das Kanalsystem in das DGM „eingebrannt“, d.h., dass alle DGM-Rasterzellen, unter denen ein Kanal verläuft, der hydrologisch relevant ist, um einige Meter eingetieft werden. Damit wird das Netz bei der Entwässerung durch das DGM berücksichtigt. Ähnlich kann auch mit Gehsteigkanten oder Gebäuden verfahren werden - hier werden DGM-Zellen erhöht, um den Oberflächenabfluss durch Gebäude zu verhindern. Inwieweit das DGM bearbeitet werden kann und muss, hängt von der Datengrundlage ab. Grundsätzlich ist aber festzustellen, dass diese Vorarbeit sinnvoll ist, und eine genauere Abbildung der Realität im Modell zulässt.

Die Verknüpfung zwischen Oberfläche und Entwässerungssystem erfolgt über die Kanalknoten und das aufbereitete DGM. Hierzu wird in QGIS über mehrere Schritte die entwässernde Fläche für jeden Knoten bestimmt.
Die entstandene Teileinzugsgebietskarte wird dann mit der Oberflächenkarte verschnitten. Damit werden die knotenbezogenen Teileinzugsgebiete in knotenbezogene Teileinzugsgebiete mit homogener Oberfläche aufgeteilt. Der assoziierte Oberflächenabfluss der homogenen Flächen wird dann direkt an die Knoten geleitet und der eigentlich stattfindende Abfluss zwischen Flächen vernachlässigt. Die entstandene Zuordnung zeigt Abb. 1b. Hier sieht man die Vereinfachung im Vergleich zu Abb. 1a, wo der Abfluss über verschiedene Teileinzugsgebiete geleitet wird, bevor er den Knoten erreicht. Diese Vereinfachung hat nur vernachlässigbare Auswirkungen auf den Gesamtabfluss, beschleunigt die Modellerstellung aber erheblich (Krebs 2016).

Die eigentliche Erstellung der SWMM-Input-Datei kann mithilfe von GDAL-Bibliotheken (Geospatial Data Abstraction Library, Rasterdaten) oder OGR (Simple Features Library, Vektordaten) erfolgen. Dazu steht z. B. swmmr (Leutnant und Doering 2020) in R (R Core Team 2017) oder in Python (Python Software Foundation 2020a) das QGIS SWMM PLUGIN (Oslandia 2018) für die Konvertierung von ShapeDateien zu SWMM-Input-Dateien zur Verfügung. Niederschlags- und Klimadaten müssen entsprechend der Vorgaben (Rossman 2010) vorbereitet werden.

Die Visualisierung, Nachbearbeitung und Evaluierung von Simulationsergebnissen kann auf vielfältige Art erfolgen. Es empfiehlt sich, Ergebnisse aus SWMM zu exportieren oder Ergebnisdateien (Binärformat) für die Weiterverarbeitung direkt auszulesen - z. B. in Python mit swmmtoolbox (Python Software Foundation 2020b). In Python oder R kann dann auch die Berechnung von Statistiken für die Modellgüte erfolgen. Die SWMM GUI bietet selbst nur rudimentäre Werkzeuge zur Visualisierung von Ergebnissen.

Zur Modellkalibrierung können Open-Source-Softwarepakete wie z.B. PEST (Brunner et al. 2012) verwendet werden. Alternativ gibt es eine Reihe implementierter Optimierungsalgorithmen in Python oder R, die Verwendung finden können (z. B. NSGAII (Deb et al. 2002)). Dabei muss allerdings oft die Schnittstelle zwischen Modell und Optimierer - Auslesen der Ergebnisse, Berechnung der Zielfunktionen, Einlesen neuer Parameterwerte in das Modell - geschrieben werden. Aller- 


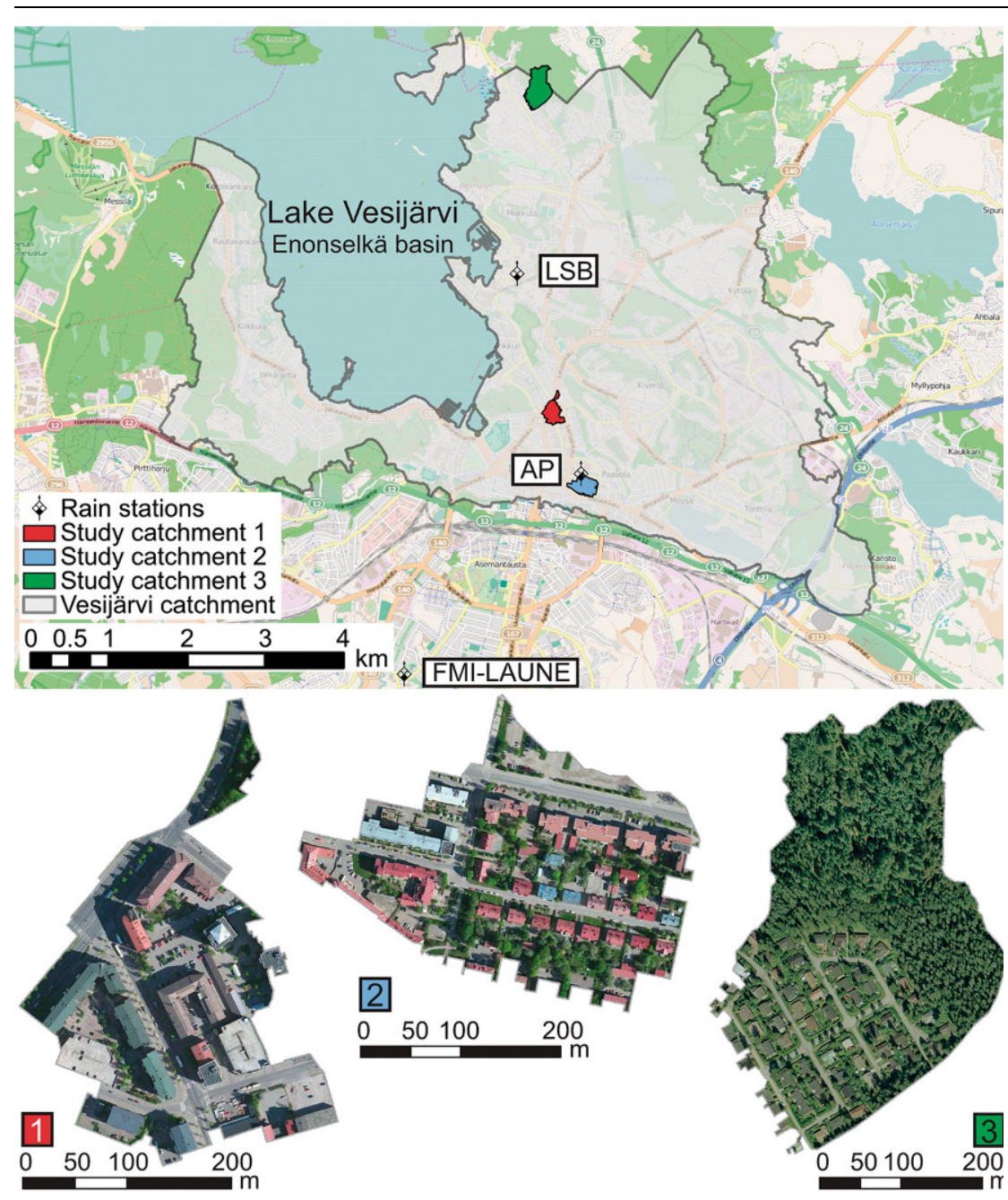

Abb. 2 Das Einzugsgebiet Vesijärvi der Stadt Lahti, die drei Teileinzugsgebiete und deren Lage im Gesamteinzugsgebiet (modifiziert von Krebs 2016)

dings können auch vorhandene Pakete wie z. B. swmmr (Leutnant and Doering 2020) mit Optimierungsalgorithmen verknüpft werden. Ebenfalls in R steht mit RSWMM (Steinberg 2015) ein lauffähiger Optimierer zur Verfügung, der auf NSGAII basiert.

\section{Anwendungsbeispiel}

Die Anwendung der beschriebenen Methodik soll hier am hydrologischhydraulischen Niederschlags-AbflussModell für die Stadt Lahti, Finnland, erläutert werden (Krebs 2016). Die Stadt Lahti (104.000 Einwohner) liegt in Südfinnland $\left(60,9^{\circ} \mathrm{N}, 25,6^{\circ} \mathrm{E}\right)$ und ist geprägt von feuchtem Kontinentalklima (durchschnittliche Lufttemperatur $4,1^{\circ} \mathrm{C}$ und durchschnittlicher Jahresniederschlag $633 \mathrm{~mm})$.

Ein Großteil der bebauten Stadtfläche wird mit einem Trennsystem in den See Vesijärvi entwässert. Für dieses Gebiet wurde im Jahr 2016 ein Niederschlags-Abfluss-Modell erstellt, das folgende Kriterien erfüllt: (i) hohe räumliche Auflösung zur Evaluierung von naturnahen Regenwasserbewirtschaftungsmaßnahmen, (ii) Kalibrierung und Regionalisierung über beprobte Teileinzugsgebiete, (iii) Verwendung von Open-Source-Produkten für eine breite Zugänglichkeit.

Im betrachteten Einzugsgebiet wurde seit 2013 der Regenwasserabfluss von drei Teileinzugsgebieten gemessen und auf Schmutzstoffe beprobt (Abb. 2). Diese Einzugsgebiete unterscheiden sich in Bebauungs- und Versiegelungsgrad und sollten als Grundlage für die Kalibrierung und Regionalisierung des Gesamteinzugsgebietsmodells dienen.

Die Erhebung der Datengrundlage ergab, dass beschreibende Oberflächendaten in hoher Genauigkeit ver- fügbar waren. Neben Gebäuden und Straßenflächen gab es auch eine Definition der Oberflächen (z. B. Asphalt, Pflastersteine, Schotter) für alle öffentlichen versiegelten Flächen. Allerdings waren keine Daten zu Parkplätzen und Einfahrten auf Grundstücken verfügbar. Diese Flächen können ca. $30 \%$ der urbanen Versiegelung ausmachen (Krebs et al. 2013). Daher wurden diese Flächen aus Luftbildern digitalisiert und in den Datensatz nachgetragen. Ein DGM stand mit einer Auflösung von $2 \times 2 \mathrm{~m}$ zur Verfügung. Der Kanalkataster wies große Lücken auf, vor allem bei kleineren Rohrdurchmessern und dazugehörigen Einlässen. Vorrangig betroffen waren davon Wohnsiedlungen, deren Grundstücksentwässerung nur unzureichend dokumentiert war. Daher wurde folgende Vorgehensweise gewählt:

1. In-situ-Datenerhebung für die drei Teileinzugsgebiete für eine möglichst genaue Modellgrundlage.

2. Räumliche Modellauflösung über homogene Oberflächeneigenschaften.

3. Kalibrierung der Teileinzugsgebietsmodelle mit vorhandenen hydrometeorologischen Daten.

4. Analyse der Auswirkungen aufgrund geringerer Auflösung des Entwässerungsnetzes und assoziierten Modellvereinfachungen auf den simulierten Abfluss in den drei Teileinzugsgebieten.

5. Entwicklung einer Regionalisierungsstrategie zur Parametrisierung des Gesamteinzugsgebiets.

Ziel dieser Schritte war zum einen, die Auswirkungen auf die Modellergebnisse zu evaluieren, wenn Teile des Kanalnetzes - in diesem Fall Rohre mit kleinen Durchmessern - nicht modelliert werden und stattdessen der Abfluss an der Oberfläche stattfindet. Zum anderen sollte eine umsetzbare Strategie entwickelt werden, wie Informationen aus der Kalibrierung der Teileinzugsgebiete auf das Gesamtgebiet übertragen werden können. Eine Modellvereinfachung wirkt sich auf die Simulationsergebnisse aus. Allerdings stellt sich die Frage, wie groß der Einfluss quantitativ ist und bis zu welchem Rahmen er akzeptabel ist. Dazu wurden zahlreiche Szenarien in Bezug auf die Datengrundlage modelliert. Die Ergebnisse ließen den Schluss zu, dass sich die Modellergebnisse einer Simulation unter Berücksichtigung von Leitungen mit einem 


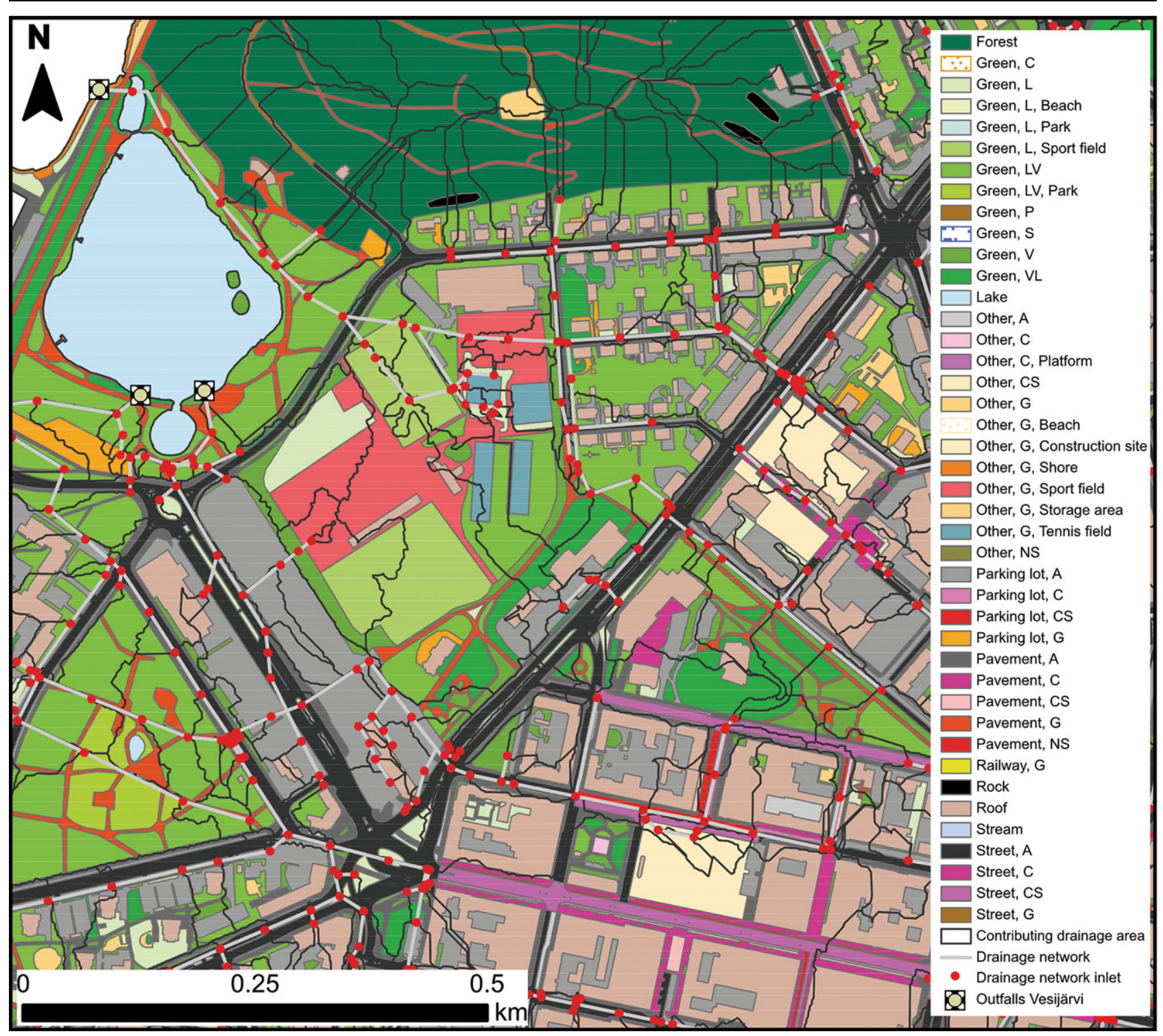

Abb. 3 Ausschnitt des erstellten SWMM-Modells mit unterschiedlichen Oberflächen (modifiziert von Krebs 2016)

Mindestdurchmesser von $300 \mathrm{~mm}$ im Vergleich zu einem „all Pipes“-Modell nur unwesentlich verschlechtern (Krebs et al. 2014). Durch die Modelldiskretisierung in Teileinzugsgebiete mit homogenen Oberflächeneigenschaften konnten kalibrierte Parameterwerte aus den Teileinzugsgebieten direkt für das Gesamtgebiet übernommen werden. Die Teileinzugsgebiete wurden dazu unabhängig voneinander kalibriert trotzdem ergaben sich sehr ähnliche Parameterwerte für gleiche Oberflächen. (Abb. 3).

Die generelle Datenaufbereitung für das Modell wurde mit einem geografischen Informationssystem erledigt. Zum Einsatz kam hier vorwiegend QGIS, zum Datenmanagement wur- de ARCGIS verwendet. Wie in der Methodik beschrieben, wurden alle verfügbaren und vor Ort erhobenen Daten in einen Datensatz verschnitten. Das DGM wurde lediglich durch das unterirdische Entwässerungssystem ergänzt, um Fließpfade korrekt abzubilden. Über die Knoten des Entwässerungsnetzes und die DGM wurde die Oberfläche mit dem Kanalnetz verknüpft. Die Haltungsdaten mussten um den Anfangs- und Endknoten ergänzt werden, um eine Verknüpfung zwischen Knoten und Haltungen zu gewährleisten.

Die erstellten Dateien (subcatchments, nodes, und links) wurden mithilfe eines Scripts, geschrieben in Perl (Wall 2007), zu einer SWMM-Input-
Datei konvertiert. Die Nachbereitung der Ergebnisse wurde zum Teil in Microsoft Excel und zum Teil mit Python durchgeführt. Hier wurden Statistiken zur Modellsensitivität berechnet und Abflusskurven dargestellt. Die drei Teileinzugsgebiete wurden mit RSWMM kalibriert. Hierzu wurde der Evolutionsalgorithmus NSGAII (Deb et al. 2002) verwendet.

Funding Open access funding provided by Graz University of Technology.

Open Access Dieser Artikel wird unter der Creative Commons Namensnennung 4.0 International Lizenz veröffentlicht, welche die Nutzung, Vervielfältigung, Bearbeitung, Verbreitung und 
Wiedergabe in jeglichem Medium und Format erlaubt, sofern Sie den/die ursprünglichen Autor(en) und die Quelle ordnungsgemäß nennen, einen Link zur Creative Commons Lizenz beifügen und angeben, ob Änderungen vorgenommen wurden.
Die in diesem Artikel enthaltenen Bilder und sonstiges Drittmaterial unterliegen ebenfalls der genannten Creative Commons Lizenz, sofern sich aus der Abbildungslegende nichts anderes ergibt. Sofern das betreffende Material nicht unter der genannten Creative Commons Lizenz steht und die betreffende Handlung nicht nach gesetzlichen
Vorschriften erlaubt ist, ist für die oben aufgeführten Weiterverwendungen des Materials die Einwilligung des jeweiligen Rechteinhabers einzuholen.

Weitere Details zur Lizenz entnehmen Sie bitte der Lizenzinformation auf http://creativecommons.org/licenses/ by/4.0/deed.de.
Berndtsson, R., Niemczynowicz, J. (1988): Spatial and temporal scales in rainfall analysis-Some aspects and future perspectives. J Hydrol 100(1-3): 293-313. https://doi.org/10.1016/ 0022-1694(88)90189-8

Brunner, P., Doherty, J., Simmons, C.T. (2012): Uncertainty assessment and implications for data acquisition in support of integrated hydrologic models. Water Res R 48(7). https:// agupubs.onlinelibrary.wiley.com/doi/abs/10. 1029/2011WR011342

Cristiano, E., ten Veldhuis, M.-C., van de Giesen, N. (2017): Spatial and temporal variability of rainfall and their effects on hydrological response in urban areas-a review. Hydrol Earth Syst Sci 21(7): 3859-3878. https://doi.org/10. 5194/hess-21-3859-2017

Deb, K., Pratap, A., Agarwal, S., Meyarivan, T (2002): A fast and elitist multiobjective genetic algorithm: NSGA-II. Evolutionary Computation, IEEE Transactions 6(2): 182-197. https://doi. org/10.1109/4235.996017

Environmental Systems Research Institute (ESRI) (2012): ArcGIS Release 10.

Haase, D., Nuissl, H. (2007): Does urban spraw drive changes in the water balance and policy? The case of Leipzig (Germany) 1870-2003. Landscape Urban Plan 80(1-2): 1-13. https://doi.org/ 10.1016/j.landurbplan.2006.03.011

Huber, W.C., Dickinson, R.E. (1988): Storm Water Management Model Manual, Version 4 User's Manual, Athens, Georgia, USA, US EPA Huong, H.T.L., Pathirana, A. (2013): Urbanization and climate change impacts on future urban flooding in Can Tho city, Vietnam. Hydrol Earth Syst Sci 17(1): 379-394. https://doi.org/10.5194/ hess-17-379-2013

Krebs, G., Kokkonen, T., Valtanen, M., Setälä, H., Koivusalo, H. (2014): Spatial resolution considerations for urban hydrological modelling. J Hydrol 512: 482-497. https://doi.org/10.1016/j. jhydrol.2014.03.013

Krebs, G., Rimpiläinen, U.-M., Salminen, 0. (2013): How does Imperviousness develop and affect runoff generation in an urbanizing waters- hed? Fennia-International Journal of Geography 191(2): 143-159. https://doi.org/10.11143/ 7794

Krebs, G. (2016): Spatial Resolution and Parameterization of an Urban Hydrological Model: Requirements for the Evaluation of Low Impact Development Strategies at the City Scale. Dissertation. Aalto University, Finnland. https:// aaltodoc.aalto.fi/handle/123456789/20293. Zugriffsdatum 15.4.2020.

Leopold, L.B. (1968): Hydrology for Urban Planning-A Guidebook on the Hydrologic Effects of Urban Land Use. US Department of the Interior Washington DC, USA

Leutnant, D., Doering, A. (2020): swmmr: R Interface for US EPA's SWMM. https:/ / github.com/ dleutnant/swmmr. Zugriffsdatum 15.4.2020.

Maier, R., Krebs, G., Pichler, M., Muschalla, D., Gruber, G. (2020): Spatial rainfall variability in urban environments-high-density precipitation measurements on a city-scale. Water 2020 12(4), 1157. https://doi.org/10.3390/w12041157 Oslandia (2018): QGIS swmm plugin. https:// github.com/Oslandia/qgis-swmm. Zugriffsdatum 15.4.2020.

Python Software Foundation (2020a): Python Language Reference. http://www.python.org. Zugriffsdatum 15.4.2020.

Python Software Foundation (2020b): swmmtoolbox. https://pypi.org/project/swmmtoolbox/ Zugriffsdatum 15.4.2020.

QGIS Entwicklungsteam (2020): QGIS Geographisches Informationssystem. Open Source Geospatial Foundation Projekt. http://qgis. osgeo.org. Zugriffsdatum 15.4.2020.

R Core Team (2017): R: A language and environment for statistical computing, Vienna, Austria R Foundation for Statistical Computing. http:// www.r-project.org/

Rawls, W.J., Ahuja, L.R., Brakensiek, D.L., Shirmohammadi, A. (1992): Infiltration and Soil Water Movement. In: Maidment D R (Hrsg), Handbook of Hydrology. McGraw-Hill, 5.1-5.51, New York, USA
Rossman, L.A. (2010): Storm Water Management Model User's Manual, Version 5.0. US EPA National Risk Management Research Laboratory, Cincinnati, Ohio, USA

Rossman, L.A. (2015): Storm Water Management Model User's Manual Version 5.1. US EPA National Risk Management Research Laboratory, Cincinnati, Ohio, USA

Schilling W (1991): Rainfall data for urban hydrology: what do we need? Atmos Res 27(1-3): 5-21. https://doi.org/10.1016/0169-8095(91) 90003-F

Schueler, T.R., Fraley-McNeal, L., Cappiella, K. (2009): Is Impervious Cover Still Important? Review of Recent Research. J Hydrol Eng 14(4): 309-315. https://doi.org/10.1061/(ASCE)10840699(2009) 14:4(309)

Singh, V.P., Woolhiser, D.A. (2002): Mathematical Modeling of Watershed Hydrology. J Hydrol Eng 7(4): 270-292. https://doi.org/10.1061/ (ASCE) 1084-0699(2002)7:4(270)

Steinberg, P.D. (2015): RSWMM: Autocalibration for EPA Stormwater Management Model (SWMM) version 5. https://github.com/ PeterDSteinberg/RSWMM. Zugriffsdatum 15.4.2020.

Wall, L. (2007): Programming is Hard, Let's Go Scripting ... https://www.perl.com/pub/2007/ 12/06/soto-11.html/. Zugriffsdatum 15.4.2020.

Wilby, R.L. (2008): Constructing climate change scenarios of urban heat island intensity and air quality. Environ Plann B 35(5): 902-919. https:// doi.org/10.1068/b33066t

Zoppou, C. (2001): Review of urban storm water models. Environ Modell Softw 16(3): 195-231. https://doi.org/10.1016/S1364-8152(00)00084-0

Hinweis des Verlags Der Verlag bleibt in Hinblick auf geografische Zuordnungen und Gebietsbezeichnungen in veröffentlichten Karten und Institutsadressen neutral. 